\title{
UVALVIT: A tool for droplet mobility control and valving
}

\author{
Nikolaos Vourdas, Konstantinos Dalamagkidis, and Vassilis N. Stathopoulos \\ School of Technological Applications, Technological Educational Institute of Sterea Ellada, Psahna, Evia, Greece, 34400
}

\begin{abstract}
Active control of droplet mobility through low cost tools is highly desirable in applications entailing microfluidics, Lab-on-Chip devices and pertinent technologies. Here, we present the design concepts of a versatile, low cost tool for dynamic droplet mobility manipulation, employing a scheme with backpressure application. Initially sticky open- or closed- channel fluidics with hydrophobic, porous walls are rendered slippery with the application of backpressure through the porous walls. Deliberate control of backpressure directs the wetting phenomena to the desired state. Operation parameters, and control system considerations are presented. Ultra-low backpressure values, are needed for channels with small cross-sections, which in turn are compatible with ultra-low energy demands.
\end{abstract}

\section{Introduction}

Starting from the early 90 s a series of outstanding advances in microfluidics technology have been performed [1, 2], enabling the research of fluid flows in the micro- and nano- scale [3], and inseminating a wide range of applications from chemical and biological analysis [4-7], to micro and nano synthesis routes [8]. Pertaining market analysis have shown the overall Compound Annual Growth Rate (CAGR), is expected to grow up to $\sim 25 \%$ from 2010 to 2016 , while the unit cost is decreasing to well below $€ 5$ (see ref. [9] and references therein). The key for further development and exploitation of these technologies includes larger-scale integration and autonomy, while a particular associated emerging market includes the use of coupled components.

In most cases, crucial functions are realized exogenously, for example the fluid flow is adjusted exogenously from typical large-scale pumps, e.g. syringe pumps in most cases. Implementation of such large scale components diminish the portability of such devices and hinders further utilization. As such, development and integration of active or passive pumps and valves has thus attracted a lot of attention lately [10-13] especially for the case of droplet based microfluidics. Several actuation and valving technologies have been proposed, including electric, thermal, optic and acoustic. All of these technologies induce heat into the droplets affecting their temperature and therefore, are not suitable for handling temperature sensitive bio particles such as cells and bacteria [14]. On the other hand, magnetics-related schemes necessitate the use of magnetic media, which introduce complexity and increase costs.

Recently alternative pneumatic-assisted schemes have been proposed for droplet actuation and mobility manipulation. Mild gas pressure/flow is applied under a porous medium [15-17], or hole [18] on top of which a droplet is situated. The pressure balance at the liquid/solid interface is changed, thus imposing respective mobility variations, and found applications in wetting control upon impingement [19] and in valving inside open-channel fluidics [20]. The presence and conservation of gas pockets resided in the solid-liquid interface and the significance of pressure gradient below and over the liquid phase has been highlighted, also in other studies [21-23].

In this work we present the design of an integrated tool, for controlling the mobility of plugs inside open- or closed-channel fluidics. The heart of the tool is a low-cost disposable fluidic with porous hydrophobic walls, that may facilitate the application of backpressure. The construction material is ceramic, exhibiting high robustness, chemical inactivity, mechanical stability and high temperature stability. With the application of backpressure, gas pockets evolve at the solid-liquid (S-L) interface, with tunable pressure and volume. With this the $\mathrm{S}-\mathrm{L}$ surface area is decreasing, the receding contact line is partially detached, while additional, anisotropic Laplace pressures appear, along with asymmetric bubbles. The collapsing of the gas pockets introduce additional forward wave, assisting the liquid movement. Through deliberate backpressure control, the above mentioned phenomena, are tuned towards the desired direction. The design concepts and considerations are presented in this work.

\section{Experimental Section}

The basic operational principle for a closed channel fluidic is depicted in Fig.1(a). A honeycomb-like structure is needed. The central channel will be used as

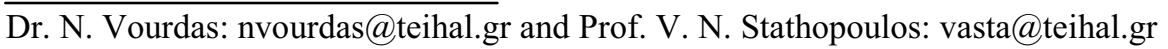




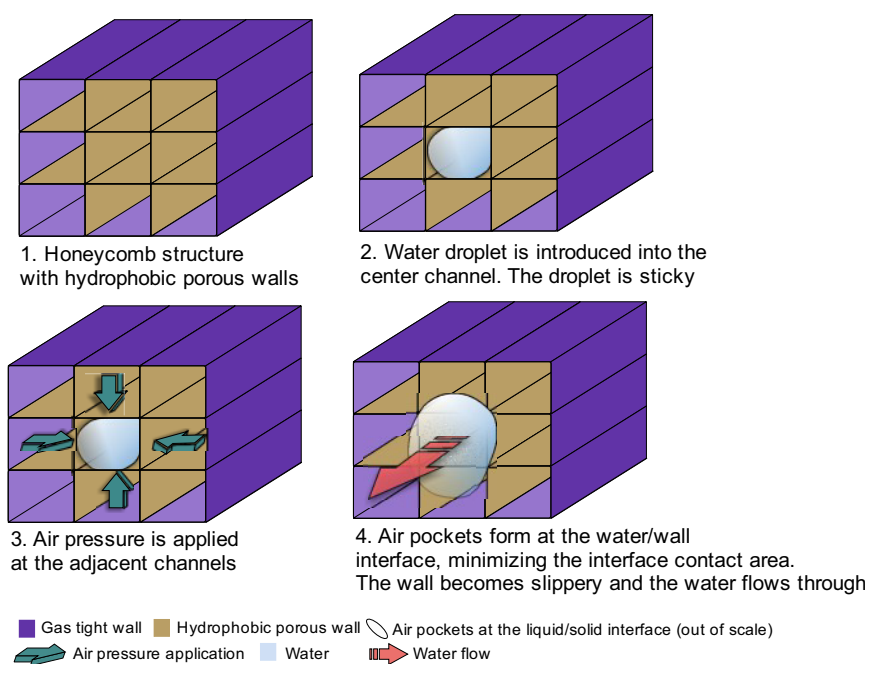

(a)

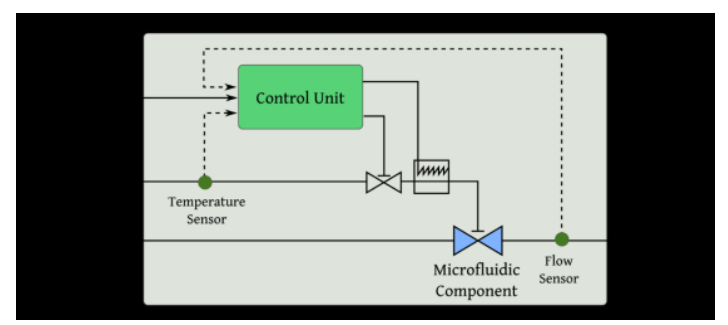

(b)

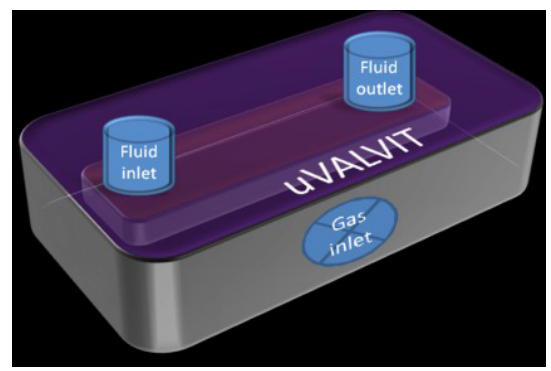

(c)

Figure 1. (a) Schematic of the operation principle for the integrated pneumatic-actuated component for the fluid flow control. (b) Logic flowchart of the component (c) Envisaged schematic of the integrated device, uVALVIT.
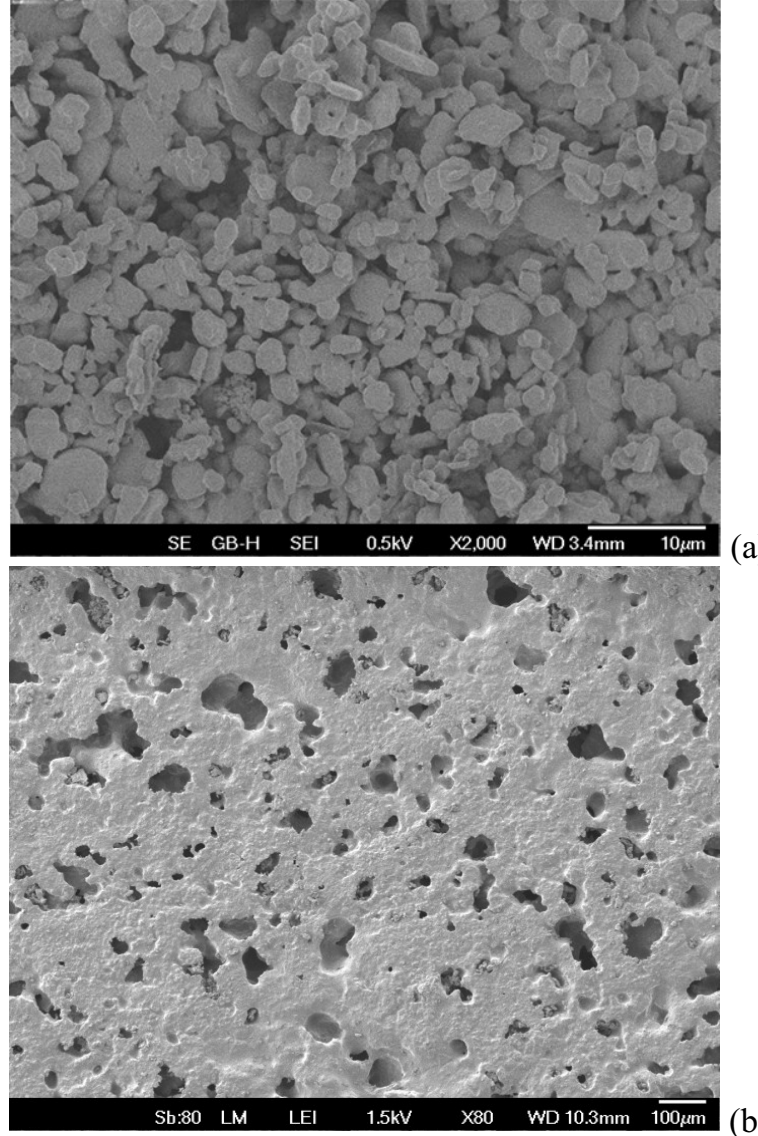

(a)

Figure 2. SEM images of the porous surfaces. (a) Porous surface prepared from shaping and sintering of primary particles, exhibiting micro-sized protrusions. (b) Porous surface from extrusion of a paste with suitable viscosity, exhibiting holes (see Ref. [15]).

the fluidic, in which the liquid plug will flow. The peripheral channels will facilitate the backpressure application. All walls are hydrophobic and porous, with the exception of the outer, which have been sealed tight.

Initially the fluidic walls are sticky, and therefore the water plug inside the channel is pinned; the valve is in "off" position. With the application of backpressure application through the peripheral channels, gas pockets evolve at the S-L interfaces, triggering the plug depinning and thus setting the valve in "on" position.

The sticky to slippery transition is reversible, in that when the backpressure is off, the plug sticks back again to the channel, thus setting the valve in the "off" position.

The simplified logic flowchart is given in Fig.1(b), and the envisaged final device in Fig.1(c).

\subsection{Fluidic Fabrication}

Different approaches may be followed in order to fabricate the fluidic with the porous walls, such as casting, extrusion, pressing followed by sintering, or 3D printing. Up to now single-axis pressing and extrusion have been used to deliver the adequate porous surfaces. The respective SEM images are given in Fig. 2. Based on the primary particles and the thermo-mechanical pretreatment different porous characteristics may be attained, tailored to the specifications for each application.

\subsection{Surface Functionalization}

The as-formed ceramic structure is highly hydrophilic and water absorbing. The surfaces are rendered hydrophobic - not superhydrophobic- by means of dry [24-26] or wet chemical processing [27]. Initially water droplets are sticky; sliding angle measurements are given hereafter. Reagents, measuring tools and preparation particularities may be found elsewhere [15]. 

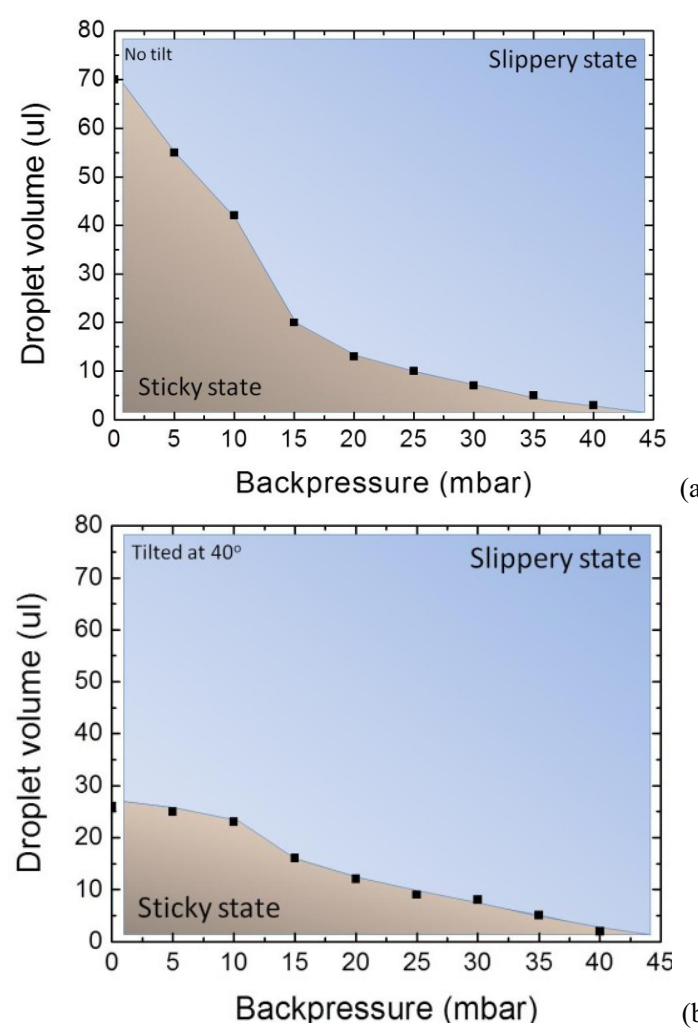

(b)

Figure 3. Effect of the backpressure on the wetting state of a porous surface with holes tilted at (a) $0^{\circ}$, and (b) $40^{\circ}$ (see Ref. [15]).

\section{Results and discussion}

\subsection{Open surfaces}

As a first step the mobility manipulation on open surfaces has been demonstrated. In Fig. 3 we present the effect of backpressure on the mobility state of porous surfaces exhibiting micro-holes as in Fig. 2b. For example the surface is inherently sticky for a 55 ul droplet (Fig. 3a).

However with an application of ultra-low backpressure of 5 mbar the surface is rendered slippery. Upon removal of backpressure the surface becomes sticky back again. In any case backpressure values lower than 45 mbar are needed for the sticky to slippery transition. This is compatible with ultra-low energy demands [15].

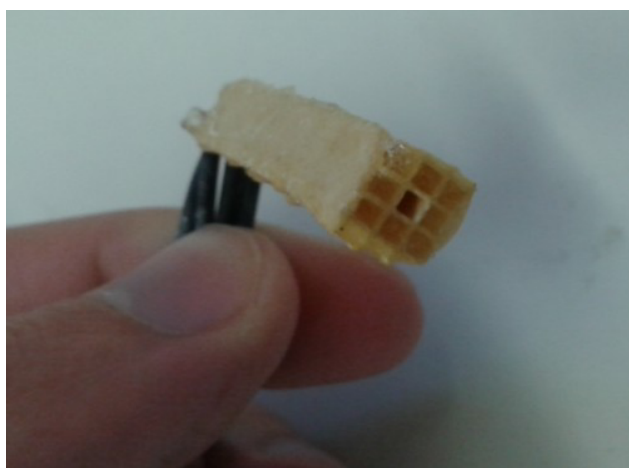

Figure 4 Photograph of a closed-channel fluidic, amenable for plug mobility manipulation by means of backpressure.

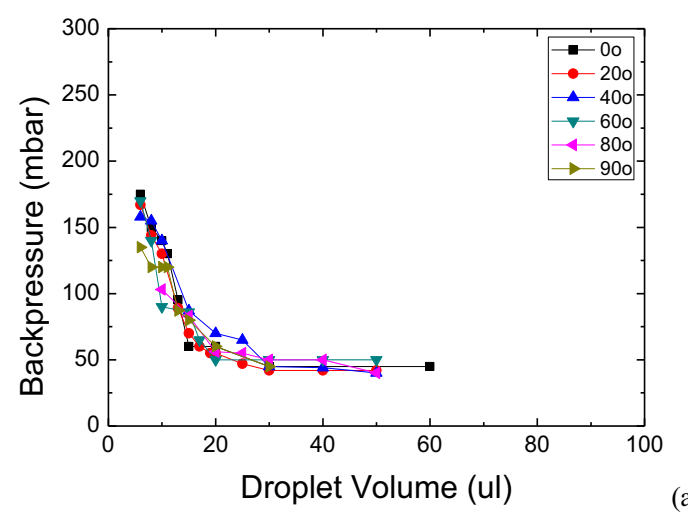

(a)

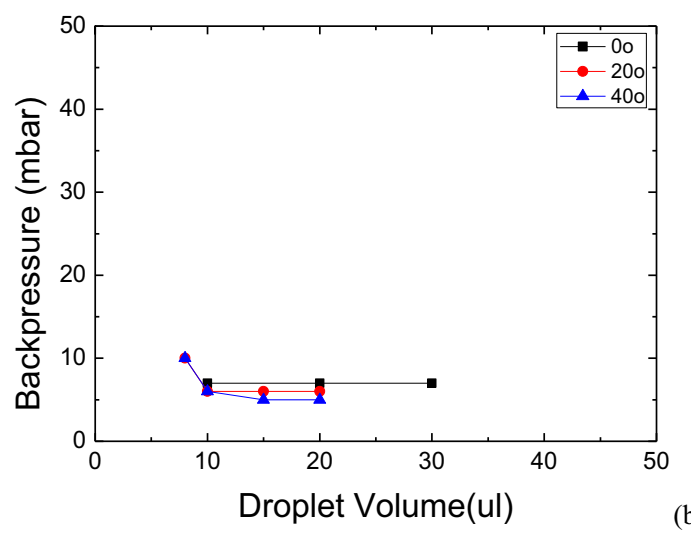

Figure 5. Effect of the backpressure on the wetting state of open-channel fluidics with porous surface exhibiting holes vs. droplet volume and tilt angle: (a) $1.5 \times 1.5 \mathrm{~mm}^{2}$, (b) $0.8 \times 0.8$ $\mathrm{mm}^{2}$.

\subsection{Closed-channel fluidics}

In Fig. 4 we present a snapshot of a closed-channel fluidic amenable for plug mobility manipulation by means of backpressure.

\subsection{Open-channel fluidics}

In Fig. 5 we present the operation conditions for openchannel fluidics with dimensions $1.5 \times 1.5 \mathrm{~mm}^{2}$ (Fig. 5a) and $0.8 \times 0.8 \mathrm{~mm}^{2}$ (Fig. $5 \mathrm{~b}$ ). The effect of droplet volume and tilt angle decreases as the channel dimensions are lowered [20]. For the case depicted in Fig. 5 b, ultra-low backpressure values up to 10 mbar are needed to induce the "off" to "on" transition, regardless the tilt angle and the droplet volume. Again this is compatible to ultra-low energy demands.

\subsection{Open-channel fluidics}

The development of the electronics is a 3-stage process:

1. In the breadboard prototyping phase, the basic connections between the components is established and tested. This provides also a platform for testing the code as well as for evaluating different design alternatives and components in isolation. 
2. The Proto-I stage involves the production of a prototype board that can be used for integration testing, debugging problems and refining the design, as well as for running a first series of experiments. At this stage all the components are present and the system is built to enable the full range of the envisioned functionality.

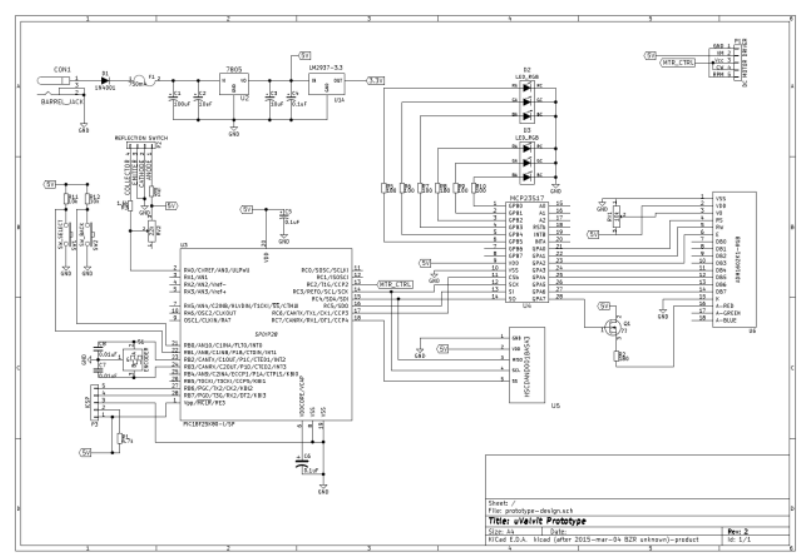

Figure 6. Schematic of the uVALVIT prototype control system.

3. The Proto-II stage involves the production of a small batch of boards incorporating all the needed changes identified in the previous phases. These boards will be small size and externally manufactured and will be used both for further experimentation with the platform as well as in dissemination activities.

The schematic that is the basis for the first two stages of the development has been completed and is shown in Fig. 6.

\section{Conclusions}

We presented the design concepts and fabrication of a versatile tool for dynamic droplet mobility manipulation. The tool is designed for both open- and closed channel fluidics. The heart of the tool is a ceramic, porous fluidic, enabling the application of backpressure through the fluidic walls. The inherently sticky walls are rendered slippery when backpressure is applied at the rear side of the channel walls. Depending on the porous characteristics and channel dimensions ultra low backpressure values are needed to induce the transition from the sticky to a slippery state.

\section{References}

[1] A. Manz, N. Graber, and H. M. Widmer, Sensors and Actuators: B. Chemical 1 (1-6), 244 (1990).

[2] G. M. Whitesides, Nature 442 (7101), 368 (2006).

[3] T. M. Squires, and S. R. Quake, Reviews of Modern Physics 77 (3), 977 (2005).

[4] J. El-Ali, P. K. Sorger, and K. F. Jensen, Nature 442 (7101), 403 (2006).

[5] N. Vourdas et al., Microelectronic Engineering 85 (5), 1124 (2008).
[6] D. Moschou et al., Sensors and Actuators B: Chemical 199 (0), 470 (2014).

[7] E. Mavraki et al., Procedia Engineering 25 (0), 1245 (2011).

[8] H. Song, D. L. Chen, and R. F. Ismagilov, Angewandte Chemie 45 (44), 7336 (2006).

[9] C. D. Chin, V. Linder, and S. K. Sia, Lab on a chip 12 (12), 2118 (2012).

[10] A. C. Glavan et al., Lab on a chip 13 (15), 2922 (2013).

[11] K. Ellinas, A. Tserepi, and E. Gogolides, Microfluid Nanofluid 7 (3), 1 (2014).

[12] A. Ghosh et al., Lab on a chip 14 (9), 1538 (2014).

[13] K. Tsougeni et al., Lab on a Chip - Miniaturisation for Chemistry and Biology 10 (4), 462 (2010).

[14] N. T. Nguyen, in 3rd European Conference on Microfluidics-Microfluidics 2012 (SHF 2012, Heidelberg, 2012), Vol. uFLU12, pp. 241.

[15] N. Vourdas, C. Ranos, and V. N. Stathopoulos, RSC Advances 5 (42), 33666 (2015).

[16] V. N. Stathopoulos, and N. Vourdas, in The 8th International Conference on Material Technologies and Modeling, MMT 2014 (2014), pp. 4.

[17] N. Vourdas, and V. N. Stathopoulos, in Technical Proceedings of the 2014 NSTI Nanotechnology Conference and Expo, NSTI-Nanotech 2014 (2014), Vol. 1, pp. 220.

[18] J. N. Tan, W. Y. L. Ling, and A. Neild, Applied Physics Express 6 (7), 077301 (2013).

[19] N. Vourdas, A. Tserepi, and V. N. Stathopoulos, Applied Physics Letters 103 (11), 111602 (2013).

[20] N. Vourdas, K. Dalamagkidis, and V. Stathopoulos, RSC Advances (2015) DOI: 10.1039/C5RA21263D.

[21] T. M. Schutzius et al., Nature 527 (7576), 82 (2015).

[22] J. Snoeijer, P. Brunet, and J. Eggers, Physical Review E 79 (3), 036307 (2009).

[23] J. de Ruiter et al., Nat Phys 11 (1), 48 (2014).

[24] N. Vourdas, A. Tserepi, and E. Gogolides, Nanotechnology 18 (12), 125304 (2007).

[25] E. Gogolides et al., International Journal of Nanomanufacturing 6 (1), 152 (2010).

[26] N. E. Vourdas et al., International Journal of Nanotechnology 6 (1-2), 196 (2009).

[27] A. Milionis et al., Materials and Manufacturing Processes (just-accepted) (2015) DOI:

10.1080/10426914.2015.1059443. 